\title{
Reconstrução do ligamento cruzado cranial em cães, associado ou não ao sulfato de condroitina
}

[Cranial cruciate ligament reconstruction in dogs associated or not to chondroitin sulfate]

\author{
F. Biasi ${ }^{1 *}$, S.C. Rahal ${ }^{2}$, R.S. Volpi $^{3}$, J.L. Sequeira ${ }^{2}$ \\ ${ }^{1}$ Pós-graduando - Faculdade de Medicina Veterinária e Zootecnia - UNESP - Botucatu, SP \\ ${ }^{2}$ Faculdade de Medicina Veterinária e Zootecnia - UNESP - Botucatu, SP \\ ${ }^{3}$ Faculdade de Medicina - UNESP - Botucatu, SP
}

\begin{abstract}
RESUMO
Avaliou-se o efeito da reconstrução do ligamento cruzado cranial, associado ou não ao sulfato de condroitina, na evolução da osteoartrite induzida experimentalmente em cães. Vinte cães hígidos, sem raça definida, machos e fêmeas, com peso corpóreo entre 19 e $25 \mathrm{~kg}$, foram submetidos à desmotomia do ligamento cruzado cranial. Trinta dias após, foram separados em dois grupos de 10 animais. Um grupo foi submetido à reconstrução do ligamento cruzado com uso de aloenxerto de ligamento patelar congelado, $\mathrm{o}$ outro não. Trinta e um dias após a desmotomia, cada grupo foi dividido em dois subgrupos de cinco animais. Um recebeu sulfato de condroitina, o outro não. Os cães foram avaliados clínica e radiograficamente antes da desmotomia e aos 30,60 e 90 dias após a desmotomia. No último momento foram realizados exames macro e microscópico. Nos cães submetidos somente à desmotomia e tratados com sulfato de condroitina houve redução na progressão das alterações ósseas, ao exame radiográfico. A reconstrução do ligamento cruzado cranial melhorou a função do membro e, quando associada ao sulfato de condroitina, houve melhor resposta. Não houve diferença entre os subgrupos quanto aos exames macro e microscópico.
\end{abstract}

Palavras-chave: cão, ligamento cruzado, osteoartrite, sulfato de condroitina

\begin{abstract}
The effect of cranial cruciate ligament reconstruction, associated or not to chondroitin sulfate, on the evolution of experimentally induced osteoarthritis in dogs was studied. Twenty healthy mixed dogs, weighing between 19 and $25 \mathrm{~kg}$ were submitted to cranial cruciate desmotomy. Thirty days later, the animals were divided into two groups with ten dogs each. One was submitted to cranial cruciate ligament reconstruction using frozen patellar tendon allograft and the other received no surgical treatment. Thirty one days after desmotomy, each group was divided into two subgroups with five animals each. One subgroup for each group received chondroitin sulfate and the other received no medical treatment. The dogs were evaluated clinically and radiographically before desmotomy, 30, 60 and 90 days after desmotomy, and at this moment, accomplished gross and microscopical exams. In dogs submitted to only desmotomy, chondroitin sulfate reduced radiographically the progression of osteoarthritic bone changes. Cranial cruciate ligament reconstruction improved dog's gait, however, when associated with chondroitin sulfate, the limb function improved even quicker. There were no statistical differences between subgroups at gross and histological evaluation.
\end{abstract}

Keywords: dog, cruciate ligament, osteoarthritis, chondroitin sulfate

Recebido para publicação em 10 de novembro de 2003

Recebido para publicação, após modificações, em 4 de julho de 2004

*Endereço para correspondência (mailing address)

Rua Sebastião Chapp Galindo, 50/701 - Bloco 1

Jardim do Lago

86061-570 - Londrina, PR

E-mail: biasif@yahoo.com 


\section{INTRODUCÃO}

A ruptura do ligamento cruzado cranial é uma lesão freqüente nos cães, e a restauração da função normal do joelho é difícil de ser obtida, mesmo após tratamento (Järvinen et al., 1995). O procedimento cirúrgico, com o objetivo de estabilizar a articulação e reduzir a progressão da osteoartrite (Rooster et al., 2001; Sandman e Harari, 2001), é amplamente recomendado para cães de médio a grande porte. Animais acima de $15 \mathrm{~kg}$ de peso corpóreo dificilmente melhoram a função do membro quando não submetidos à reconstrução ligamentar (Vasseur, 1984). Em 85 a $90 \%$ dos casos operados a osteoartrite progride, independentemente da técnica cirúrgica adotada. Pearson (1971), Heffron e Campbell (1979) e Vasseur e Berry (1992) relataram progressão da doença articular degenerativa em joelho de cães mesmo após a reconstrução do ligamento cruzado cranial.

O efeito condroprotetor dos glicosaminoglicanos polissulfatados já foi demonstrado experimentalmente (Hannan et al., 1987). O sulfato de condroitina (glicosaminoglicano monossulfatado) tem sido investigado em estudos bioquímicos graças ao seu papel na fisiologia da cartilagem articular (Pipitone, 1991). Entre as propriedades condroprotetoras destaca-se a ação inibitória de enzimas de degradação da cartilagem, (Diaz et al., 1996) e entre as propriedades condroestimuladoras, o aumento da síntese de proteoglicanos pelos condrócitos (Beale et al., 1990).

A terapia da osteoartrite deve ser direcionada para a analgesia, controle da inflamação, limitação aos danos dos tecidos articulares e tentativas de cicatrização da cartilagem articular (Lees, 1999). Canapp et al. (1999) e Souza et al. (1999) observaram melhora na função do membro após tratamento com sulfato de condroitina associado a glicosamina ou com precursores de glicosaminoglicanos em modelo de sinovite ou osteoartrite sem instabilidade articular, respectivamente. Melo et al. (2000) verificaram melhora macroscópica em modelo no qual se induziu a osteoartrite pela ruptura do ligamento cruzado cranial, submetido ao tratamento com sulfato de condroitina. Esse último modelo de indução da osteoartrite foi descrito em vários estudos (Marshall, 1969; Pond e Nuki, 1973; Johnson e Johnson, 1993; May, 1994), e se caracteriza por instabilidade articular.

O trabalho teve por objetivo avaliar o efeito da reconstrução do ligamento cruzado cranial, associado ou não ao sulfato de condroitina, na evolução da osteoartrite induzida pela ruptura do ligamento cruzado cranial.

\section{MATERIAL E MÉTODOS}

Vinte cães hígidos, sem raça definida, machos e fêmeas, entre um e três anos de idade e pesos entre $19 \mathrm{e} 25 \mathrm{~kg}$, foram submetidos à desmotomia do ligamento cruzado cranial baseado no modelo Pond e Nuki (Pond e Nuki, 1973). Após 30 dias, os animais foram separados em dois grupos de 10. Os do grupo I não receberam tratamento cirúrgico e os do grupo II foram submetidos à reconstrução do ligamento cruzado cranial com uso de aloenxerto de tendão patelar congelado. A técnica cirúrgica procedeu-se por meio de artrotomia parapatelar medial, inspeção dos meniscos e remoção dos remanescentes do ligamento cruzado cranial. Foram realizados túneis na tíbia e no fêmur para a passagem do enxerto, fixado com grampo de aço e com parafuso de interferência, respectivamente na tíbia e no fêmur. Após os procedimentos cirúrgicos (desmotomia ou reconstrução do ligamento), foi administrada buprenorfina ${ }^{1}$ $(10 \mu \mathrm{g} / \mathrm{kg}$, via subcutânea, de 12 em 12 horas, durante cinco dias), e após a reconstrução ligamentar, aplicou-se ampicilina benzatina ${ }^{2}$ $(20 \mathrm{mg} / \mathrm{kg}$, via subcutânea, de $24 \mathrm{em} 24$ horas, durante cinco dias).

No $31^{\circ}$ dia, o grupo I foi dividido em subgrupos A e B e o grupo II em subgrupos C e D, com cinco cães cada. Os subgrupos $\mathrm{A}$ e $\mathrm{C}$ receberam $240 \mathrm{mg}$ de sulfato de condroitina, via subcutânea, a cada sete dias, totalizando oito injeções. Os subgrupos $\mathrm{B}$ e $\mathrm{D}$ não receberam tratamento médico.

Os cães foram avaliados por exames clínico e radiográfico antes da desmotomia (M1), e aos 30 (M2), 60 (M3) e 90 dias (M4) após a

\footnotetext{
1 TENGESIC ${ }^{\circledR}$ - Schering Plough S.A. - Estrada dos Bandeirantes, 3091 - Rio de Janeiro - RJ.

${ }^{2}$ OPTACILIN $1000^{\circledR}$ - Byk Quim. e Farm. Ltda. - Av. Casa Grande, 850 - Diadema - SP.
} 
desmotomia. A avaliação clínica incluiu exames da locomoção e da estabilidade crânio-caudal da tíbia em relação ao fêmur. A claudicação foi classificada em escores: 0, normal; 1, claudicação somente após o exercício; 2, claudicação ao caminhar e correr; 3 , claudicação ao caminhar e elevação do membro ao correr; e 4, impotência funcional do membro. A estabilidade articular foi graduada em escores: 0 , ausente; 1, movimento de gaveta cranial presente, porém limitado - discreta instabilidade articular; e 2, movimento de gaveta acentuado de intensidade semelhante à observada após a desmotomia do ligamento cruzado cranial. As radiografias foram obtidas nas posições craniocaudal, mediolateral e tangencial, todas avaliadas de acordo com Vasseur e Berry (1992).

Os cães foram submetidos à eutanásia 90 dias após a desmotomia, por anestesia geral barbitúrica seguida da administração intravenosa de cloreto de potássio. As lesões macroscópicas foram graduadas de acordo com Volpi (1989). Para a superfície cartilaginosa da tróclea femoral os escores foram: 0, ausência de irregularidades; 1, discretas irregularidades superficiais; 2, discretas irregularidades superficiais e úlcera discreta ou osteófito discreto; e 3, irregularidades intensas e/ou úlcera intensa e/ou intenso osteófito. Para a superfície cartilaginosa dos côndilos femorais lateral e medial os escores foram: 0 , ausente; 1, perda de brilho; 2, irregularidades finas e/ou úlceras discretas; e 3, irregularidades grosseiras e/ou ulcerações profundas. A cor da membrana sinovial foi avaliada separadamente. Para o exame microscópico, as amostras, colhidas da tróclea femoral e dos côndilos femorais lateral e medial, foram fixadas em formalina neutra tamponada a $10 \%$, desmineralizadas em solução de ácido nítrico a 12,5\%, desidratadas em álcool e embebidas em parafina. Os cortes de 4 a $5 \mu \mathrm{m}$ de espessura, corados com hematoxilina-eosina e tricrômio de Masson, foram examinados ao microscópico óptico. Para cada região da superfície articular, as lesões foram classificadas em escores: 0, ausência de lesões; 1, descamação, formação de fendas e alteração da linha nível; 2, desorganização das colunas de condrócitos, atrofia da cartilagem, membrana fibrosa sobre a superfície articular, nichos de condrócitos; e 3, invasão de vasos na cartilagem, cistos, ulceração e formação de osteófitos. Os resultados foram submetidos ao teste estatístico não-paramétrico Friedman, Kruskal-Wallis e Mann-Witney, segundo indicações de Siegel (1975), considerando-se as diferenças estatísticas quando $\mathrm{P}<0,05$.

\section{RESULTADOS E DISCUSSÃO}

O modelo utilizado foi eficaz em induzir lesões compatíveis com osteoartrite ou doença articular degenerativa, já descritas por Marshall (1969), Pond e Nuki (1973), Johnson e Johnson (1993) e May (1994).

Nos cães dos subgrupos C e D houve melhora da função do membro quando comparadas à dos subgrupos A e B no M3 (Tab. 1). Isto era esperado e reforça a importância da reconstrução do ligamento cruzado cranial em cães com peso acima de $15 \mathrm{~kg}$, como citado por Vasseur (1984). A técnica cirúrgica adotada para a reconstrução do ligamento cruzado foi satisfatória com relação ao retorno à função do membro na maioria dos casos, confirmando os resultados de Rooster et al. (2001) e Sandman e Harari (2001).

Não houve diferença significativa na locomoção entre os cães dos subgrupos A e B, após o tratamento com sulfato de condroitina. Canapp et al. (1999) verificaram melhora na locomoção, após 12 dias de medicação com sulfato de condroitina associado à glicosamina, em sinovite induzida quimicamente na articulação rádiocárpica em cão. Souza et al. (1999) relataram melhora na função do membro após a abrasão cartilaginosa do sulco da tróclea femoral concomitantemente ao uso de precursores de glicosaminoglicanos por 15 dias de tratamento. Entretanto, vale salientar que ambos os modelos não resultaram em instabilidade articular. Os resultados do grupo I indicaram que o sulfato de condroitina não teve efeito significativo na melhora da locomoção dos cães que apresentavam os joelhos instáveis.

O subgrupo $\mathrm{C}$ teve melhora significativa em M3 quando comparado ao $\mathrm{M} 2$, e o subgrupo D não apresentou melhora significativa de M2 para M3 (Tab. 1). O subgrupo $\mathrm{C}$ teve melhora mais rápida da função do membro quando comparado ao subgrupo D. Este resultado reforça o efeito benéfico dos glicosaminoglicanos, em relação à função locomotora do membro, no tratamento da 
osteoartrite, como relatado por Canapp et al. (1999) e Souza et al. (1999), quando não se tem instabilidade articular, pois o grupo II teve menor grau de movimento de gaveta cranial que o grupo I (Tab. 2).

Tabela 1. Mediana da função locomotora do membro posterior esquerdo* de cães, segundo os grupos, subgrupos e momentos

\begin{tabular}{lccccc}
\hline \multirow{2}{*}{ Grupo } & \multirow{2}{*}{ Subgrupo } & \multicolumn{4}{c}{ Momento } \\
\cline { 3 - 6 } & & M1 & M2 & M3 & M4 \\
\hline \multirow{2}{*}{ G1 } & $\mathrm{A}$ & $0 \mathrm{Aa}$ & $3 \mathrm{Ba}$ & $2 \mathrm{Ba}$ & $2 \mathrm{Ba}$ \\
\multirow{2}{*}{ G2 } & $\mathrm{B}$ & $0 \mathrm{Aa}$ & $3 \mathrm{Ba}$ & $3 \mathrm{Ba}$ & $3 \mathrm{Ba}$ \\
& $\mathrm{C}$ & $0 \mathrm{Aa}$ & $3 \mathrm{Ba}$ & $2 \mathrm{Cb}$ & $1 \mathrm{Ab}$ \\
& $\mathrm{D}$ & $0 \mathrm{Aa}$ & $3 \mathrm{Ba}$ & $1 \mathrm{Bb}$ & $0 \mathrm{Ab}$ \\
\hline
\end{tabular}

* Função locomotora medida em escores: 0, normal; 1, claudicação somente após o exercício; 2, claudicação ao caminhar e correr; 3 , claudicação ao caminhar com elevação do membro ao correr; 4 , impotência funcional do membro.

Valores seguidos por letras maiúsculas distintas na mesma linha, ou por letras minúsculas distintas na mesma coluna, diferem entre si $(\mathrm{P}<0,05)$.

G1: desmotomia com reconstrução do ligamento cruzado cranial; GII: sem reconstrução; A: associada à condroitina; B: sem condroitina; C: com condroitina; D: sem condroitina; M1: pré-desmotomia; M2: 30 dias pós-desmotonia; M3: 60 dias pós-desmotonia; M4: 90 dias pós-desmotonia.

Tabela 2. Mediana da estabilidade do joelho esquerdo* de cães segundo os grupos e momentos

\begin{tabular}{lcccc}
\hline \multirow{2}{*}{ rrupo } & \multicolumn{4}{c}{ Momento } \\
\cline { 2 - 5 } & M1 & M2 & M3 & M4 \\
\hline GI & $0^{\mathrm{a}}$ & $2^{\mathrm{a}}$ & $2^{\mathrm{a}}$ & $2^{\mathrm{a}}$ \\
GII & $0^{\mathrm{a}}$ & $2^{\mathrm{a}}$ & $1^{\mathrm{b}}$ & $1^{\mathrm{b}}$ \\
\hline * Estabilidade
\end{tabular}

de gaveta cranial presente, porém limitado, e discreta instabilidade articular; 2 , movimento de gaveta acentuado de intensidade semelhante à observada após a desmotomia do cruzado cranial. Valores seguidos por letras distintas diferem entre si $(\mathrm{P}<0,05)$.

GI: desmotomia com reconstrução do ligamento cruzado cranial; GII: sem reconstrução; M1: pré-desmotomia; M2: 30 dias pós-desmotomia; M3: 60 dias pós-desmotomia; M4: 90 dias pós-desmotomia.

Ao exame radiográfico, observaram-se osteófitos, entesiófitos e erosão do osso subcondral no joelho esquerdo dos cães. Houve progressão das alterações ósseas no período pósoperatório em todos os subgrupos (Tab. 3), o que está de acordo com Pearson (1971), Heffron e Campbell (1979) e Vasseur e Berry (1992), que relataram progressão da doença articular degenerativa mesmo nos cães submetidos à cirurgia para estabilização articular. Ela foi mais acentuada no subgrupo B (sem cirurgia e sem condroitina), quando comparada à do subgrupo
A (sem cirurgia e com condroitina), ao final do período de observação (M4). Esse fato pode demonstrar o efeito protetor dos glicosaminoglicanos, como citado por Diaz et al. (1996), Hannan et al. (1987), Beale et al. (1990), Pipitone (1991) e Lees (1999), mesmo com a instabilidade articular presente nesse modelo de osteoartrite.

Tabela 3. Mediana da alteração óssea pelo exame radiográfico ${ }^{*}$ de cães, segundo os grupos, subgrupos e momentos

\begin{tabular}{|c|c|c|c|c|c|}
\hline \multirow{2}{*}{ Grupo } & \multirow{2}{*}{ Subgrupo } & \multicolumn{4}{|c|}{ Momento } \\
\hline & & M1 & M2 & M3 & M4 \\
\hline \multirow{2}{*}{ G1 } & A & $0 \mathrm{Aa}$ & $2 \mathrm{Ba}$ & $4 \mathrm{Ca}$ & $7 \mathrm{Da}$ \\
\hline & B & $0 \mathrm{Aa}$ & $1 \mathrm{Aa}$ & $10 \mathrm{Ba}$ & $14 \mathrm{Cb}$ \\
\hline \multirow{2}{*}{ G2 } & $\mathrm{C}$ & $0 \mathrm{Aa}$ & $2 \mathrm{Ba}$ & $6 \mathrm{Ca}$ & $6 \mathrm{Ca}$ \\
\hline & $\mathrm{D}$ & $0 \mathrm{Aa}$ & $2 \mathrm{Ba}$ & $3 \mathrm{Ba}$ & $10 \mathrm{Ca}$ \\
\hline
\end{tabular}

moderada; 3 , grave. A soma dos escores de cada lesão presente no joelho indicou a intensidade da osteoartrite, segundo Vasseur e Berry (1992).

Valores seguidos por letras maiúsculas distintas na mesma linha, ou por letras minúsculas distintas na mesma coluna, diferem entre si $(\mathrm{P}<0,05)$.

G1: desmotomia com reconstrução do ligamento cruzado cranial; GII: sem reconstrução; A: associada à condroitina; B: sem condroitina; C: com condroitina; D: sem condroitina; M1: pré-desmotomia; M2: 30 dias pós-desmotonia; M3: 60 dias pós-desmotonia; M4: 90 dias pós-desmotonia.

Ao exame macroscópico, os joelhos dos cães do subgrupo A mostraram pigmentação alaranjada na membrana sinovial, com menor intensidade do que a dos cães do subgrupo $\mathrm{B}$, o que pode indicar menor grau de sinovite. Entretanto, quando os quatro subgrupos foram comparados entre si, não houve diferença significativa para as lesões macroscópicas avaliadas (Tab. 4), ao contrário do verificado por Melo et al. (2000), que observaram menor incidência de alterações macroscópicas no joelho de cães com osteoartrite induzida pela ruptura do ligamento cruzado cranial, submetidos ao tratamento com sulfato de condroitina.

Ao exame microscópico, notaram-se alterações compatíveis com osteoartrite, tais como descamação e fenda cartilaginosas, desorganização das colunas de condrócitos, nichos de condrócitos e úlcera de cartilagem (Fig. 1). Não houve diferença significativa quando comparados os quatro subgrupos entre si. A não significância, ao exame microscópico, pode ter relação com o local da colheita do material, já que se obtiveram apenas três 
amostras de cada joelho, na superfície articular do fêmur.

Tabela 4. Mediana dos exames macro ${ }^{*} \mathrm{e}$ microscópico $^{* *}$ da superfície cartilaginosa da tróclea femoral $(\mathrm{T})$, do côndilo femoral medial (M) e do côndilo femoral lateral (L) de cães, segundo os grupos, subgrupos e momentos

\begin{tabular}{|c|c|c|c|c|c|}
\hline \multirow{2}{*}{ Exame } & \multirow{2}{*}{ Grupo } & \multirow{2}{*}{ Subgrupo } & \multicolumn{3}{|c|}{ Região } \\
\hline & & & $\mathrm{T}$ & $\mathrm{M}$ & $\mathrm{L}$ \\
\hline \multirow{4}{*}{ Macroscópico } & \multirow{2}{*}{ GI } & $\mathrm{A}$ & $2 a$ & $2 \mathrm{a}$ & $1 \mathrm{a}$ \\
\hline & & $\mathrm{B}$ & $3 a$ & $2 \mathrm{a}$ & $3 a$ \\
\hline & \multirow{2}{*}{ GII } & $\mathrm{C}$ & $2 \mathrm{a}$ & $2 \mathrm{a}$ & $1 \mathrm{a}$ \\
\hline & & $\mathrm{D}$ & $2 \mathrm{a}$ & $2 \mathrm{a}$ & $1 \mathrm{a}$ \\
\hline \multirow{4}{*}{ Microscópico } & \multirow{2}{*}{ GI } & A & $2 a$ & $2 \mathrm{a}$ & $0 \mathrm{a}$ \\
\hline & & B & $2 \mathrm{a}$ & $2 \mathrm{a}$ & $2 \mathrm{a}$ \\
\hline & \multirow{2}{*}{ GII } & $\mathrm{C}$ & $2 \mathrm{a}$ & $2 \mathrm{a}$ & $2 \mathrm{a}$ \\
\hline & & $\mathrm{D}$ & $3 a$ & $1 \mathrm{a}$ & $2 \mathrm{a}$ \\
\hline
\end{tabular}

* Exame macroscópico da superfície cartilaginosa da tróclea femoral em escores: 0, ausente; 1 , discretas irregularidades superficiais; 2, discretas irregularidades superficiais e úlcera discreta ou osteófito discreto; 3 , irregularidades intensas e/ou úlcera intensa e/ou intenso osteófito. Para a superfície cartilaginosa dos côndilos femorais lateral e medial em escores: 0 , ausente; 1 , perda de brilho; 2 , irregularidades finas e/ou úlceras discretas; 3 , irregularidades grosseiras e/ou ulcerações profundas.

** Exame microscópico em escores: 0, ausência de lesões; 1, descamação, formação de fendas e alteração da linha nível; 2, desorganização das colunas de condrócitos, atrofia da cartilagem, membrana fibrosa sobre a superfície articular, nichos de condrócitos; 3, invasão de vasos na cartilagem, cistos, ulceração e formação de osteófitos.

Valores seguidos por letras minúsculas distintas na coluna diferem entre si $(\mathrm{P}<0,05)$.

G1: desmotomia com reconstrução do ligamento cruzado cranial; GII: sem reconstrução; A: associada à condroitina; $\mathrm{B}$ : sem condroitina; $\mathrm{C}$ : com condroitina; D: sem condroitina; M1: pré-desmotomia; M2: 30 dias pós-desmotonia; M3: 60 dias pós-desmotonia; M4: 90 dias pós-desmotonia.

\section{CONCLUSÕES}

Nas condições do experimento, o sulfato de condroitina reduz a velocidade das alterações ósseas radiográficas compatíveis com osteboartrite induzidas experimentalmente em joelho de cães, dentro de 30 a 60 dias de tratamento. A reconstrução ligamentar associada ao sulfato de condroitina promove melhora mais rápida na função do membro quando comparada com a reconstrução isoladamente.

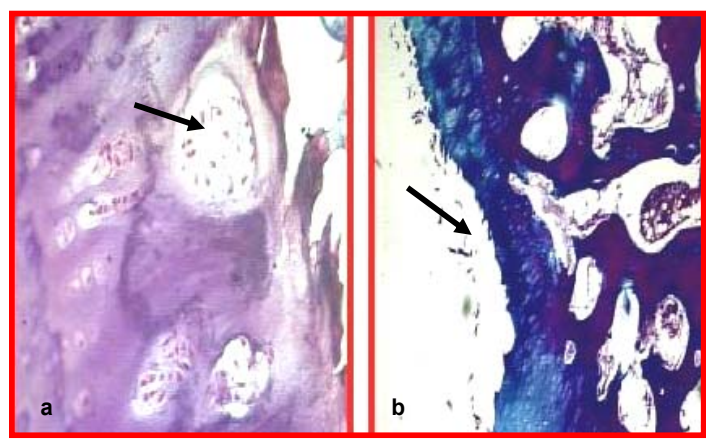

Figura 1. Cortes histológicos da superfície articular do fêmur esquerdo do cão $\mathrm{n}^{\mathrm{o}} 5$, do subgrupo B, 90 dias após a desmotomia do ligamento cruzado cranial. (a) nichos de condrócitos (seta) na cartilagem do côndilo medial (hematoxilina-eosina-400x); ulceração cartilaginosa (seta) (tricrômio de Masson-100x).

\section{REFERÊNCIAS BIBLIOGRÁFICAS}

BEALE, B.S.; GORING, R.L.; CLEMMONS, R.M. et al. Effect of semi-synthetic polysulfated glycosaminoglycan on the hemostatic mechanism in the dog. Vet. Surg., v.19, p.57, 1990.

CANAPP Jr., S.O.; McLAUGHLIN Jr., R.M.; HOSKINSON, J.J. et al. Scintigraphic evaluation of dogs with acute synovitis after treatment with glucosamine and chondroitin sulfate. Am. J. Vet. Res., v.60, p.1552-1557, 1999.

DIAZ, V.B.; FUENTS, E.P.; MARTINEZ, O.E. et al. Chondroitin sulfate (Overview). In: SALAMONE, J.C. (Ed.). Polymeric materials encyclopedia. Boca Raton: CRC, 1996. v.2, p.1262-1274.

HANNAN, N.; GHOSH, P.; BELLENGER, C. et al. Systemic administration of glycosaminoglycan polysulphate (Arteparon) provides partial protection of articular cartilage from damage produced by meniscectomy in the canine. J. Orthop. Res., v.5, p.47-59, 1987.

HEFFRON, L.E.; CAMPBELL, J.R. Osteophyte formation in the canine stifle joint following treatment for rupture of the cranial cruciate ligament. J. Small Anim. Pract., v.20, p.603-611, 1979. 
JÄRVINEN, M.; JOZSA, L.; JOHNSON, R.J. et al. Effect of anterior cruciate ligament reconstruction with patellar tendon or prosthetic ligament on the morphology of the other ligaments of the knee joint. Clin. Orthop. Relat. Res., v.311, p.176-182, 1995.

JOHNSON, J.M.; JOHNSON, A.L. Cranial cruciate ligament rupture. Pathogenesis, diagnosis and postoperative rehabilitation. Vet. Clin. North Am.: Small Anim. Pract., v.23, p.717-733, 1993.

LEES, P. Pharmacology and therapeutics of joint diseases. CPD Vet. Med., v.2, p.18-26, 1999.

MARSHALL, J.L. Periarticular osteophytes. Initiation and formation in the knee of the dog. Clin. Orthop., v.62, p.37-47, 1969.

MAY, S.A. Degenerative joint disease (osteoarthritis, osteoarthrosis, secondary joint disease). In: HOULTON, J.; COLLINSON, R. (Eds.). Manual of small animal arthrology. England: BSAVA, 1994. p.62-74.

MELO, E.G.; REZENDE, C.M.F.; GOMES, M.G. et al. Condroprotetores no tratamento da doença articular degenerativa: avaliação artroscópica da membrana sinovial e da cartilagem articular. Ciên. Anim. Bras., v.1, p.68, 2000 .

PEARSON, P.T. Ligamentous and meniscal injuries of the stifle joint. Vet. Clin. North Am.: Small Anim. Pract., v.1, p.489, 1971.

PIPITONE, V.R. Chondroprotection with chondroitin sulfate. Drugs Exp. Clin. Res., v.17, p.3-7, 1991 .
POND, M.J.; NUKI, G. Experimentally-induced osteoarthritis in the dog. Ann. Rheum. Dis., p.32, v.387-388, 1973.

ROOSTER, H.; VANGHELUVE, L.; VAN BREE, H. et al. Biomechanical properties of braided polyester tapes intended for use as intraarticular cranial cruciate ligament prostheses in dogs. Am. J. Vet. Res., v.62, p.48-53, 2001.

SANDMAN, K.M.; HARARI, J. Canine cranial cruciate ligament repair techniques: is one best? Vet. Med., v.96, p.850-856, 2001.

SIEGEL, S. Estatística não paramétrica - Para ciências do comportamento. São Paulo: McGrallHill, 1975. 350p.

SOUZA, R.L.; RAISER, A.G.; GUIMARÃES, L.D. et al. Precursores de glicosaminoglicanos na reparação articular após trauma iatrogênico no joelho de cães. Clin. Vet., v.23, p.33-38, 1999.

VASSEUR, P.B. clinical results following nonoperative management for rupture of the cranial cruciate ligament in dogs. Vet. Surg., v.13, p.243-246, 1984.

VASSEUR, P.B.; BERRY, C.R. Progression of stifle osteoarthrosis following reconstruction of the cranial cruciate ligament in 21 dogs. J. Am. Anim. Hosp. Assoc., v.28, p.129-136, 1992.

VOLPI, R.S. Padronização de modelo experimental de artropatia induzida pela imobilização contínua do joelho, em coelhos. 1989. 94f. Dissertação (Mestrado em Bases Gerais da Cirurgia e Cirurgia Experimental) Faculdade de Medicina, Universidade Estadual Paulista "Júlio de Mesquita Filho", Botucatu, SP. 